\title{
Development of real-time indoor human tracking system using LoRa technology
}

\author{
Ida Syafiza Binti Md Isa, Anis Hanani \\ Faculty of Electrical and Electronic Engineering Technology, Universiti Teknikal Malaysia Melaka, Melaka, Malaysia
}

\begin{abstract}
Industrial growth has increased the number of jobs hence increase the number of employees. Therefore, it is impossible to track the location of all employees in the same building at the same time as they are placed in a different department. In this work, a real-time indoor human tracking system is developed to determine the location of employees in a real-time implementation. In this work, the long-range (LoRa) technology is used as the communication medium to establish the communication between the tracker and the gateway in the developed system due to its low power with high coverage range besides requires low cost for deployment. The received signal strength indicator (RSSI) based positioning method is used to measure the power level at the receiver which is the gateway to determine the location of the employees. Different scenarios have been considered to evaluate the performance of the developed system in terms of precision and reliability. This includes the size of the area, the number of obstacles in the considered area, and the height of the tracker and the gateway. A real-time testbed implementation has been conducted to evaluate the performance of the developed system and the results show that the system has high precision and are reliable for all considered scenarios.
\end{abstract}

This is an open access article under the CC BY-SA license.

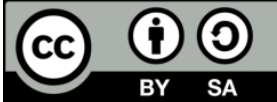

\section{Corresponding Author:}

Ida Syafiza Binti Md Isa

Faculty of Electrical and Electronic Engineering Technology, Universiti Teknikal Malaysia Melaka, Hang Tuah Jaya, 76100 Durian Tunggal Melaka, Malaysia

Email: idasyafiza@utem.edu.my

\section{INTRODUCTION}

Internet of things (IoT) is a physical device that is connected to the internet to develop interactive applications such as smart homes, smart parking systems, smart cities, and many more. The IoT also allows data sharing between devices besides enables the device to control the related system using smartphones or laptops. Besides, several studies on the energy and fog infrastructure are conducted to support the IoT demand for sending the data to the server [1]-[3] Many wireless technologies have been used to support the development of IoT networks such as Wi-Fi, Bluetooth, Zigbee, FM signal, and radio frequency identification (RFID). These technologies are often used to support the development of both indoor and outdoor localization system. However, most of the wireless technology such as Wi-Fi, Bluetooth, and Zigbee is not applicable to cover a large area [4]. Besides, they have their limitations in terms of accuracy, transmission range, and cost. For example, Wi-Fi has high accuracy but consumed high power. Meanwhile, Bluetooth has a low transmission range. Long-range (LoRa) technology is another wireless technology that has been widely used for low power large area networks (LPWAN) to support the machine to machine (M2M) and IoT application [5], [6]. LoRa is a new networking system that emphasizes low power usage and can handle several transmitters at different locations within a region by using a single receiver in the LoRa 
network [5], [6]. Besides, LoRa has a large coverage radius of up to $15 \mathrm{~km}$, hence can limit the required number of nodes to cover an environment.

The advancement of technology brings the most efficient application in the industrial and to the daily lives routine. Workplace safety is very important for every employee in the industry as they have the right to work in a safe and healthy environment. In an industrial, the employee will be exposed to an unexpected dangerous situation at the workplace such as emergencies, fire accidents, or natural disasters. Therefore, an efficient localization system is needed to locate a human when a disaster happened. Many tracking systems have been developed for various applications for instance to track objects, and human presence. Some of the developed systems utilized the global positioning system (GPS) to perform the localization due to its high accuracy of up to five meters. However, GPS is not an advisable option for indoor localization [5], [6]. Zhang et al. [7] used a sound source to locate a human in a disaster location by analyzing the changed auditory information between robot and human. However, using the robot in a complex environment is not efficient. Also, the distraction of the sound from the environment will give a significant effect on the performance of the system. Goian et al. [8], a deep learning-based human detection algorithm is proposed to locate the presence of a human in a disaster area by processing the data collected from RBG camera, thermal and wireless sensor. However, this approach has a limitation where the victim can only be identified when the victim is located on the surface. A study on human localization using radio frequency identification (RFID) is conducted in [9] to locate a human body by locating the RFID tags to the ceiling and while the RFID reader is attached to the human body. Meanwhile, in study [10] the location of the human body is located using omnidirectional vision. However, the results show that the localization precision is less at the edge of the image. Kim et al. [11] integrates vision and sound systems for real-time human localization. It shows that the proposed method is more accurate and reliable compared to the result that only used sound localization. Suzuki et al. [12] studied the human body localization using a multipleinput multiple-output ultra-wideband (MIMO-UWB) radar system. In this work, the reflected wave from the human body is extracted and analyzed. The body position is localized with an error of $20 \mathrm{~cm}$ or less by using six antennas. A cooperative passive infrared (PIR) sensor is used for human indoor localization in [13], [14], where the position is estimated based on the PIR sensor data. A wireless sensor network has also been studied in [15], [16] for real-time localization. This method estimates the location of the sensor node along with the inertial sensors worn by a person. Meanwhile, Konings et al. [17] proposed indoor human localization using passive visible light positioning.

The receiver signal strength indication (RSSI) is the most popular and simplest method that is used for both indoor and outdoor localization. This is because it does not requires additional hardware and can be found on any devices utilizing any type of wireless communication technology. RSSI works by calculating the signal strength of the packet received at the receiver. Note that, the signal strength decrease as the signal propagates away from the transmitter. Therefore, for localization, the RSSI is used by finding the approximate distances between the transmitter and receiver. Several researchers used the RSSI-based approach to indicate the signal strength of the transmitter at the receiver, for indoor and outdoor localization [5], [6], [18]-[23]. Wi-Fi is a wireless technology that is commonly used for indoor localization with the RSSI-based method due to its low cost, wide-coverage, and high efficiency [18]. Several recent studies [6], [18], [24], the authors developed an indoor localization system using Wi-Fi to improve the localization system by measuring the RSSI value. Besides, Grzechca et al. [25] integrated the use of the surveillance video and the RSSI-based method using the Wi-Fi module to identify the object in the indoor environment. Meanwhile, Gu and Ren [19] study the relationship between the RSSI value and the device location and proposed a Motion-assisted Device Tracking Algorithm to develop an energy-efficient indoor localization scheme. The proposed scheme leverage the user motions to reduce the search space to locate the target device. Meanwhile, the work in [5] considered the LoRa technology for indoor localization where the RSSI value is used as the measurement parameter in the testing. The test-bed experiment has been conducted by changing the position of the LoRa transmitter while the location of the LoRa receiver is fixed. The results show that environmental factors such as the size of the area and the distances between the LoRa transmitter and receiver can give a significant impact on the RSSI value, hence affect the positioning performance. Sadowski and Spachos [26], a test-bed implementation has been conducted to compare the accuracy and the power consumption of four wireless technologies including the Wi-Fi, Bluetooth, Zigbee and long-range wide area network (LoRaWAN) for indoor localization using RSSI-based approach. The performance of the four wireless technologies for the indoor localization are evaluated considering two scenarios related to the size of the area, where for each scenario, several distance between the transmitter and receiver is considered. The results show that, the Wi-Fi has high accuracy while the Bluetooth has the lowest power consumption. The results also show that, LoRaWAN has the utmost transmission range when maximum transmission power is used.

Int J Elec \& Comp Eng, Vol. 12, No. 1, February 2022: 845-852 
To the best of our knowledge, most of the studies only consider the size of the area and the distance between the transmitter and receiver to evaluate the performance of the developed indoor localization system. However, the impact of LoRa receiver height and the amount of the obstacles in different size of the area on the RSSI value for the indoor localization system are not taken into account. Therefore, in this study, we developed a real-time indoor human tracking system using LoRa technology using the RSSI-based approach and evaluate its performance in terms of precision and reliability in two different sizes of the area while considering the height of both LoRa receiver and transmitter and the number of obstacles for each considered area. Note that, in this system, a LoRa transmitter known as LoRa tracker will be attached to the human body. Meanwhile, a LoRa receiver will act as the LoRa gateway to determine the RSSI value based on the received signal strength packet from the LoRa tracker.

\section{RESEARCH METHOD}

\subsection{Architecture of the proposed real-time indoor human tracking system}

The architecture of the proposed real-time indoor human tracking system consists of two main parts which are a tracker (i.e. transmitter) and a gateway (i.e. receiver). The communication between the tracker and the gateway is performed wirelessly using LoRa technology. Figure 1 shows the architecture of the two main parts of the proposed real-time indoor human tracking system using LoRa communication. The tracker will act as a transmitter and is equipped with a LoRa communication module and is known as LoRa tracker. In this work, the LoRa tracker will be attached to the employee located in a building. Meanwhile, the gateway will act as a receiver and is equipped with a LoRa communication module and a computer and is known as LoRa gateway. Note that, the LoRa gateway will measure the RSSI value of the signal strength packet received from the LoRa tracker. A parallax data acquisition (PLX-DAQ) software is used at the LoRa gateway to display the details of the RSSI reading (i.e. time, the tracker's ID, and RSSI value). The details of each component used to develop the proposed system will be presented in the later subsection.

Figure 2 shows the flow chart of the developed real-time indoor human tracking system. In this system, the user located in the building will be equipped with LoRa tracker. First, the connection between the LoRa tracker and LoRa gateway is activated. Next, the LoRa tracker will send data to the LoRa gateway. If LoRa gateway received the data, then it will read the RSSI value, else the LoRa gateway will initialize the system until the transmission is succeeded. The RSSI valued obtained at the LoRa gateway will be displayed in the PLX-DAQ platform.

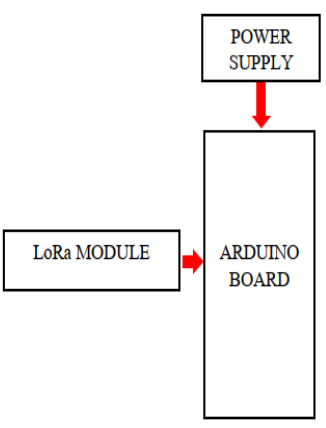

LoRa Tracker

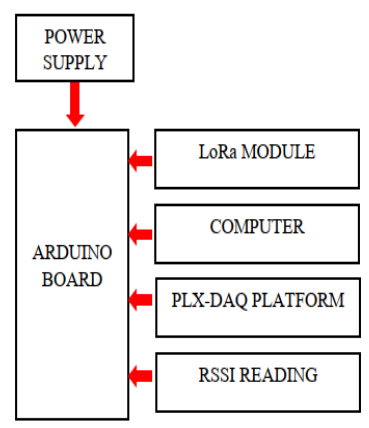

LoRa Gateway
Figure 1. Architecture of the proposed real-time indoor human tracking system

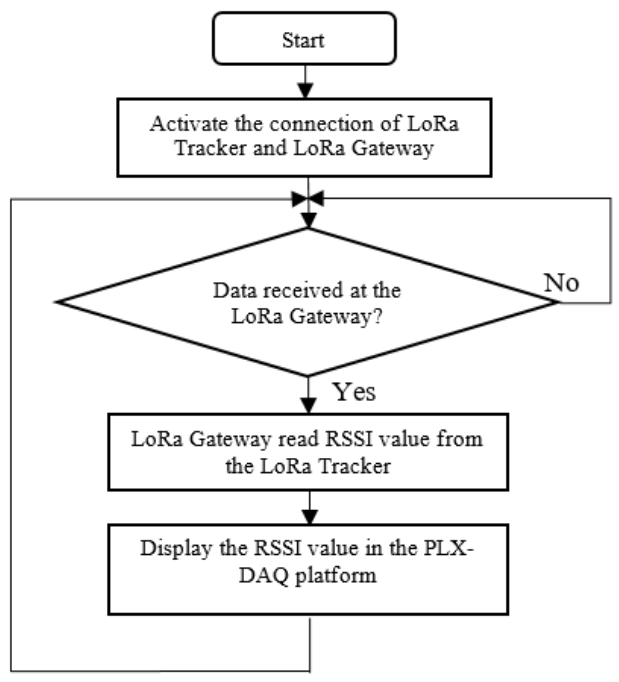

Figure 2. The flowchart of the proposed real-time indoor human tracking system

\subsection{Hardware development}

In this work, two types of LoRa trackers, LoRa tracker 1 and LoRa tracker 2, are developed using Arduino Nano and Arduino Maker-Uno, respectively. The Arduino Nano is used with the assumption that the devices will be worn on the wrist as it has a small size. Meanwhile, the Arduino Maker-Uno which has a bigger size than the Arduino Nano is used with the assumption that the device will be hung around the neck. 
The LoRa module used in this work is SX1278 which operates at $3.3 \mathrm{~V}$. The prototype of the three modules, the LoRa tracker 1, LoRa tracker 2, and LoRa gateway are as shown in Figure 3(a), Figure 3(b) and Figure 3(c), respectively.

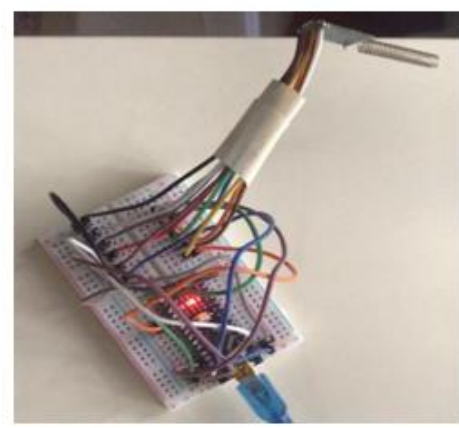

(a)

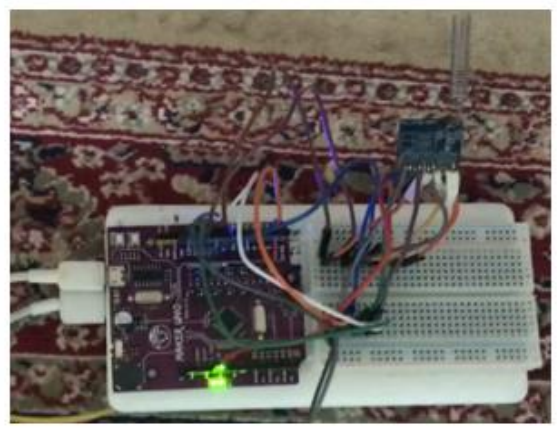

(b)

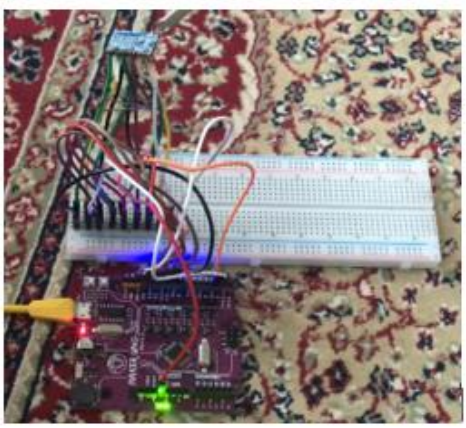

(c)

Figure 3. The prototype of (a) LoRa tracker 1, (b) LoRa tracker 2, and (c) LoRa gateway

\section{RESULTS AND DISCUSSION}

A real-time indoor human tracking and monitoring system was successfully developed and its functionality and performance have been tested. Recall that, the system consists of two parts: two transmitters (i.e. Lora tracker 1 and LoRa tracker 2) to send the data and one receiver (i.e. Lora gateway) that acts as a gateway to receive the data and calculate the RSSI value signal. The calculated RSSI value signal at the LoRa gateway will be recorded and displayed using parallax data acquisition tools (PLX-DAQ) software in Microsoft Excel. Also, the PLX-DAQ will record the time it receives data from the LoRa trackers. To display these data, serial communication is considered between the LoRa gateway and the computing by using 'Serial. Print' command with a baud rate of 9600 bits/sec. Figure 4 shows the PLX-DAQ software interface in Microsoft Excel.

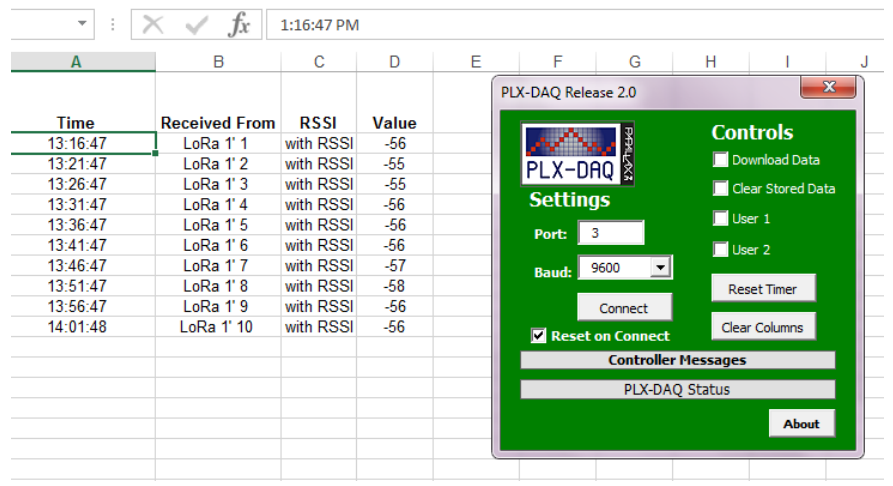

Figure 4. The PLX-DAQ interface

\subsection{Evaluation scenario}

The developed system is evaluated in terms of its precision and reliability for real-time implementation. Eight scenarios related to the size of the test-bed implementation, the number of obstacles in the test-bed implementation, and the height of the gateway for 30 minutes with 5 minutes interval are considered to test the performance of the developed system.

a. Scenario 1: Environment 1 with less obstacles

In the first scenario (i.e. scenario 1), a room size of 2.86-meter width x 2.89-meter length (i.e. environment 1 ) is considered to place the LoRa tracker 1 at a height of $22.5 \mathrm{~cm}$ from the floor as shown in Figure 5(a). This level is chosen with the assumption that the LoRa tracker 1 is placed on the wrist of the employees. Minimal obstacles such as furniture and walls which might cause interference and affect the RSSI 
signal value are considered. Note that, the considered room to place the LoRa tracker 1 is located on the first floor of the building while the LoRa gateway is placed outside the room which is the living room on the same floor.

b. Scenario 2: Environment 1 with additional obstacles

In the second scenario (i.e. scenario 2), the LoRa tracker 1 is placed at the same location as in scenario 1. However, in this scenario, more obstacles are considered in the room to study the effect on the RSSI value with the increasing obstacles as shown in Figure 5(b).

c. Scenario 3: Environment 1 with less obstacles and increased height of LoRa gateway

In the third scenario (i.e. scenario 3), the same size of the room with low obstacles as in scenario 1 is considered. However, in this scenario, the height of the LoRa gateway is increased to $63 \mathrm{~cm}$ as shown in Figure 6.

d. Scenario 4: Environment 1 with additional obstacles and increased height of LoRa gateway

In the fourth scenario (i.e. scenario 4), the same size of the room with additional obstacles as in scenario 2 is considered. However, the location of the LoRa gateway is placed at a height of $63 \mathrm{~cm}$ as shown in Figure 6.

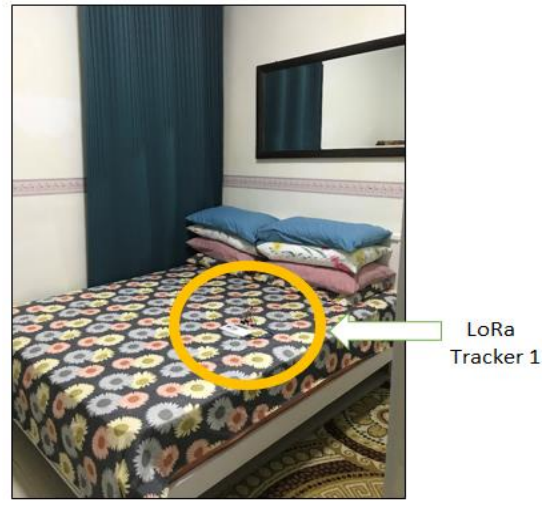

(a)

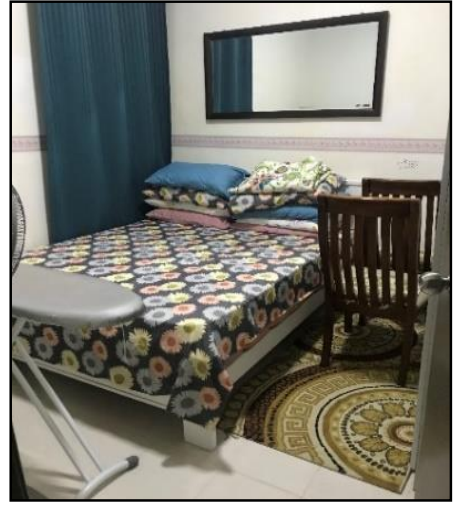

(b)

Figure 5. Location LoRa tracker 1: (a) with a minimum obstacle and (b) with additional obstacles for environment 1

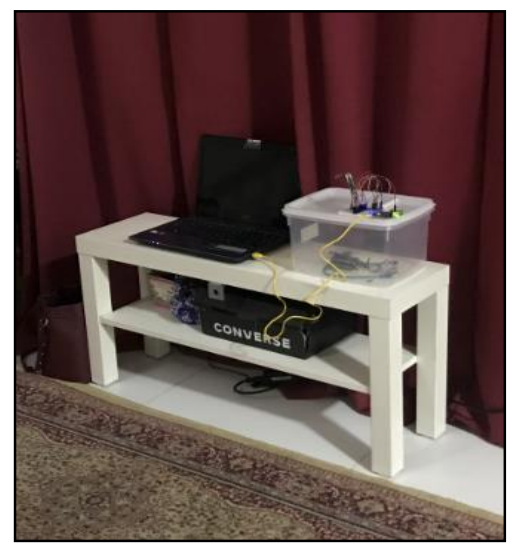

Figure 6. Location of LoRa gateway at $63 \mathrm{~cm}$ height

e. Scenario 5: Environment 2 with less obstacles

In the fifth scenario (i.e. scenario 5), a room with a size of 3.9-meter width x 4.2-meter length (i.e. environment 2) is considered to place the LoRa tracker 2 at a height of $98 \mathrm{~cm}$ from the floor as shown in Figure 7(a). The height is chosen with the assumption that the employee is wearing the identification holder around their neck. Note that, the considered room is located on the second floor of the building while the LoRa gateway is placed in the living room on the first floor. Also in scenario 5, minimum obstacles are considered. 
f. Scenario 6: Environment 2 with additional obstacles

In the sixth scenario (i.e. scenario 6), the same size of the room and the same location to place both LoRa tracker 2 and LoRa gateway as in scenario 5 are considered. However, in this scenario, additional obstacles are considered as shown in Figure 7(b).

g. Scenario 7: Environment 2 with less obstacles and increased height of LoRa gateway

In the seventh scenario (i.e. scenario 7), the same size of the room with low obstacles as in scenario 5 is considered. However, in this scenario, the height of the LoRa gateway on the first floor is increased to 63 $\mathrm{cm}$ as shown in Figure 6.

h. Scenario 8: Environment 2 with additional obstacles and increased height of LoRa gateway

In the eighth scenario (i.e. scenario 8), the same size of the room with additional obstacles as in scenario 6 is considered. However, in this scenario, the height of the LoRa gateway on the first floor is increased to $63 \mathrm{~cm}$ as shown in Figure 6.

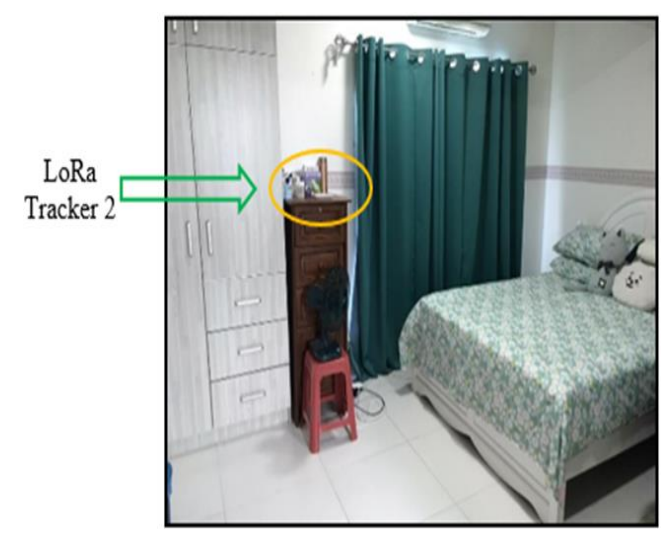

(a)

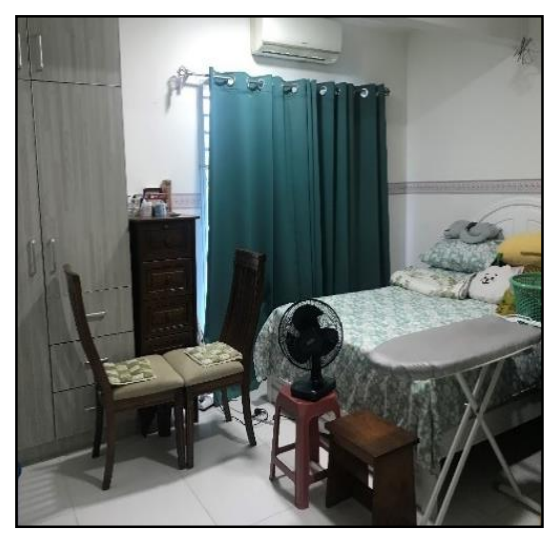

(b)

Figure 7. Location LoRa tracker 2: (a) with a minimum obstacle and (b) with maximum obstacle for an environment 2

\subsection{Results}

The value of the RSSI signal is obtained during the experiment to evaluate the performance of the LoRa communication in terms of precision and reliability, for indoor localization for human tracking system. To evaluate the reliability of the developed system, eight scenarios related to the size of the environment, the number of obstacles and the height of the gateway (i.e. receiver) has been considered during the experiment. Meanwhile, the performance of the developed system in term of precision is evaluated by running the experiment for 30 minutes with 5 minutes interval to record the value of the RSSI signal for each considered scenarios. Figure 8 shows the value of the RSSI signal for all considered scenarios for 30 minutes.

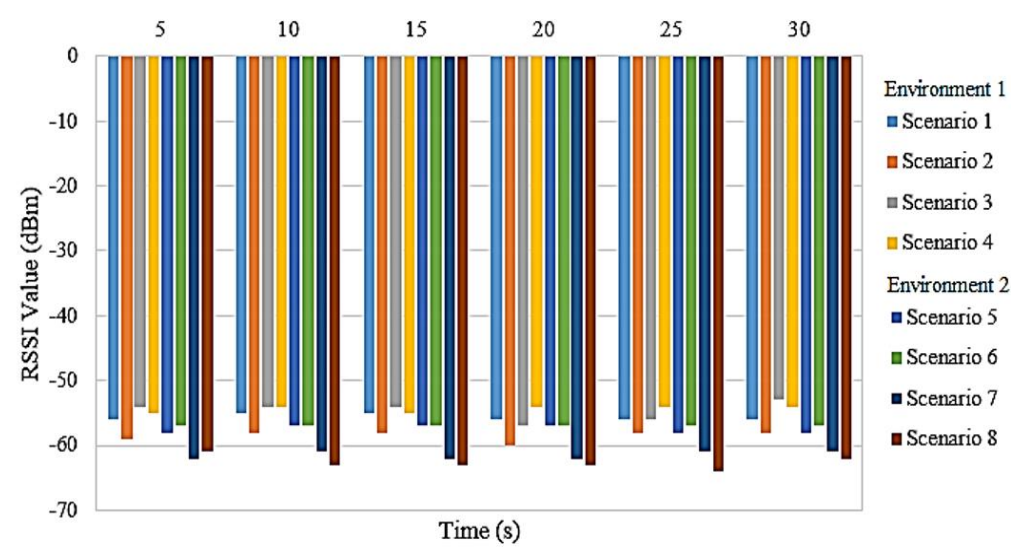

Figure 8. RSSI value of all scenarios for a 30 minutes duration 
The results show that the value of the RSSI reading for all considered scenarios for 30 minutes is almost the same. This shows that the developed system has high precision. The results also show that adding more obstacles in environment 1 reduced the RSSI value. This is due to a large number of reflections of the transmitted signal. However, for environment 2, increasing the number of obstacles does not give a significant impact on the RSSI value. This is due to the large size of room considered in environment 2 which allows the transmitted signal to propagate with less reflection. In addition, this is also due to the location of both LoRa tracker and LoRa gateway (i.e. at different building floor) which increase the distance, introduced more reflection and interference to the transmitted signal regardless of increasing the number of obstacles. Meanwhile, the results show that increasing the height of LoRa gateway located on the ground floor has increased the RSSI value for environment 1. This is mainly due to the less number of reflections of the signals off the ground, hence reduce the effects of multi-path that occurs while transmitting the signal. However, for environment 2, increasing the height of LoRa gateway reduced the RSSI value. This was found to be due to the high interference at this position between the LoRa tracker and LoRa gateway.

\section{CONCLUSION}

In this work, a real-time indoor human tracking system has been developed to monitor the location of the employees in the building using Arduino and LoRa communication technology. The RSSI signal value between the LoRa tracker ( 1 and 2 ) and the LoRa gateway is used for localization. The system is also designed to store the data at the gateway using the PLX-DAQ software tool for monitoring purposes. Several scenarios are considered including the size of the test-bed implementation, the number of obstacles in each considered test-bed implementation, and the height of the LoRa gateway to evaluate the performance of the developed system in terms of reliability. Also, the performance of the developed system is evaluated in term of precision by determining the value of the RSSI signal for all scenarios every 5 minutes interval for 30 minutes. The results indicate that increasing the number of obstacles reduced the value of RSSI when the location of the LoRa tracker and LoRa gateway is on the same floor in a building. However, the RSSI value can be increased by increasing the height of LoRa gateway as the multi-path signal effects reduced while transmitting the signal due to the reduction in the number of reflection of the transmitted signals off the ground. Also, the results show that when the LoRa tracker and the LoRa gateway are placed at different floor level in a building and a bigger room is considered, increasing the number of obstacles does not give a significant impact on the RSSI value. This is because the larger the room the smaller the amount of reflection of the transmitted signal occurred. The results show that increasing the height of LoRa gateway while locating the LoRa tracker at different floor level reduced the RSSI value as this position introduced high interferences. In addition, the results indicate that the developed system has high precision as the value of the RSSI values collected for a duration of 30 minutes are almost the same.

\section{ACKNOWLEDGEMENTS}

Authors would like to thank Centre for Research and Innovation Management (CRIM) of Universiti Teknikal Malaysia Melaka (UTeM) and Ministry of Education for supporting this research.

\section{REFERENCES}

[1] I. S. M. Isa, M. O. I. Musa, T. E. H. El-gorashi, A. Q. Lawey, and J. M. H. Elmirghani, "Energy efficiency of fog computing health monitoring applications," 2018 20th International Conference Transparent Opt. Networks, 2018, pp. 1-5, doi: 10.1109/ICTON.2018.8473698.

[2] I. S. M. Isa, T. E. H. El-Gorashi, M. O. I. Musa, and J. M. H. Elmirghani, "Energy efficient fog based healthcare monitoring infrastructure," IEEE Access, vol. 8, pp. 197828-197852, 2020, doi: h10.1109/ACCESS.2020.3033555.

[3] I. S. M. Isa, M. O. I. Musa, T. E. H. El-Gorashi, and J. M. H. Elmirghani, "Energy efficient and resilient infrastructure for fog computing health monitoring applications," in International Conference on Transparent Optical Networks, 2019, pp. 1-5, doi: 10.1109/ICTON.2019.8840438.

[4] C. Del-Valle-Soto, L. J. Valdivia, R. Velázquez, L. R. Dominguez, and J. C. L. Pimentel, "Smart campus: An experimental performance comparison of collaborative and cooperative schemes for wireless sensor network," Energies, vol. 12, no. 16, pp. 2019, doi: 10.3390/en12163135.

[5] A. Zourmand, A. L. K. Hing, C. W. Hung, and M. Abdulrehman, "Internet of things (IoT) using LoRa technology," I2CACIS 2019-Proceedings IEEE International Conference on Automatic Control and Intelligent Systems, 2019, pp. 324-330, doi: 10.1109/I2CACIS.2019.8825008.

[6] S. Sadowski and P. Spachos, "RSSI-based indoor localization with the internet of things," IEEE Access, vol. 6, pp. 30149-30161, 2018, doi: 10.1109/ACCESS.2018.2843325

[7] B. Zhang, K. Masahide, and H. Lim, "Sound source localization and interaction based human searching robot under disaster environment," 2019 SICE International Symposium Control System (SICE ISCS), vol. 1, pp. 16-20, 2019, doi: 10.23919/SICEISCS.2019.8758766.

[8] A. Goian, R. Ashour, U. Ahmad, T. Taha, N. Almoosa, and L. Seneviratne, "Victim localization in USAR scenario exploiting multi-layer mapping structure," Remote Sensors, vol. 11, no. 22, pp. 1-25, 2019, doi: 10.3390/rs11222704. 
[9] N. Pathanawongthum and P. Cherntanomwong, "Empirical evaluation of RFID-based indoor localization with human body effect," 2009 15th Asia-Pacific Conference Communication, APCC 2009, no. Apcc, 2009, pp. 479-482, doi: 10.1109/APCC.2009.5375589.

[10] J. Zhang, B. Xu, and J. Lu, "The localization algorithm of human body based on omnidirectional vision," Proceedings - 2011 6th IEEE Joint International Information Technology and Artificial Intelligence Conference, ITAIC 2011, 2011, vol. 2, pp. 172-176, doi: 10.1109/ITAIC.2011.6030303.

[11] S. W. Kim, J. Y. Lee, D. Kim, B. J. You, and N. L. Doh, "Human localization based on the fusion of vision and sound system," URAI 2011 - 2011 8th International Conference on Ubiquitous Robots and Ambient Intelligence URAI 2011, 2011, pp. 495-498, doi: 10.1109/URAI.2011.6145870

[12] T. Suzuki, K. Takizawa, and T. Ikegami, "A study on human body localization while walking in an indoor environment by using UWB signal with multiple antennas," International Symposium on Medical Information and Communication Technology, ISMICT, 2013, pp. 131-134, doi: 10.1109/ISMICT.2013.6521715.

[13] K. C. Lai, B. H. Ku, and C. Y. Wen, "Using cooperative PIR sensing for human indoor localization," 201827 th Wireless and Optical Communication Conference, WOCC 2018, pp. 1-5, 2018, doi: 10.1109/WOCC.2018.8372703.

[14] D. Yang, W. Sheng, and R. Zeng, "Indoor human localization using PIR sensors and accessibility map," 2015 IEEE International Conference on Cyber Technology in Automation, Control and Intelligent Systems, IEEE-CYBER 2015, pp. 577-581, 2015, doi: 10.1109/CYBER.2015.7288004

[15] L. Klingbeil and T. Wark, "A wireless sensor network for real-time indoor localisation and motion monitoring," Proceedings 2008 International Conference on Information Processing in Sensor Networks, IPSN 2008, 2008, pp. 39-50, 2008, doi: 10.1109/IPSN.2008.15.

[16] L. Lazos and R. Poovendran, "SeRLoc: Robust localization for wireless sensor networks," ACM Transactions on Sensor Networks, vol. 1, no. 1, pp. 73-100, 2005, doi: 10.1145/1077391.1077395.

[17] D. Konings, N. Faulkner, F. Alam, E. M. K. Lai, and S. Demidenko, "FieldLight: Device-free indoor human localization using passive visible light positioning and artificial potential fields," IEEE Sensors Journal, vol. 20, no. 2, pp. 1054-1066, 2020, doi: 10.1109/JSEN.2019.2944178.

[18] W. Xue, W. Qiu, X. Hua, and K. Yu, "Improved Wi-Fi RSSI measurement for indoor localization," IEEE Sensors Journal, vol. 17, no. 7, pp. 2224-2230, 2017, doi: 10.1109/JSEN.2017.2660522.

[19] Y. Gu and F. Ren, "Energy-efficient indoor localization of smart hand-held devices using bluetooth," IEEE Access, vol. 3, pp. 1450-1461, 2015, doi: 10.1109/ACCESS.2015.2441694.

[20] T. Jutamas, S. Pichaya, and P. Sathaporn, "Indoor wireless sensor network localization using RSSI based weighting algorithm method for short range wireless communication," in 2018 International Electrical Engineering Congress (iEECON), 2018, pp. 1-4, doi: 10.1109/IEECON.2018.8712255.

[21] N. Honma, K. Ishii, Y. Tsunekawa, H. Minamizawa, and A. Miura, "DOD-based localization technique using RSSI of indoor beacons," 2015 International Symposium on Antennas and Propagation, ISAP 2015, 2016, pp. 20-21.

[22] F. Wu, J. Xing, and B. Dong, "An indoor localization method based on RSSI of adjustable power WiFi router," Proceedings - 5th International Conference on Instrumentation and Measurement, Computer, Communication, and Control, IMCCC 2015, no. 2, 2016, pp. 1481-1484.

[23] C. Wang, R. Huang, M. Gu, and G. Xiao, "RSSI-based indoor location with circularly polarized antennas and LUDM algorithm for wireless sensor network," in 2017 Sixth Asia-Pacific Conference on Antennas and Propagation (APCAP), 2017, pp. 1-3.

[24] Y. Sasiwat, N. Jindapetch, D. Buranapanichkit, and A. Booranawong, "An experimental study of human movement effects on rssi levels in an indoor wireless network," BMEiCON 2019 - 12th Biomedical Engineering International Conference, 2019, pp. 1-5, doi: 10.1109/IMCCC.2015.313

[25] D. Grzechca, T. Wrobel, and P. Bielecki, "Indoor location and idetification of objects with video survillance system and WiFi module," Proceedings - 2014 International Conference on Mathematics and Computers in Sciences and in Industry, MCSI 2014, 2014, pp. 171-174, doi: 10.1109/MCSI.2014.52.

[26] S. Sadowski and P. Spachos, "RSSI-Based indoor localization with the internet of things," IEEE Access, vol. 6, pp. 30149-30161, 2018, doi: 10.1109/ACCESS.2018.2843325. 